\title{
Photomolecular High-Temperature Superconductivity
}

\author{
M. Buzzi $\odot,{ }^{1, *}$ D. Nicoletti $\odot,{ }^{1}$ M. Fechner, ${ }^{1}$ N. Tancogne-Dejean $\odot,{ }^{1}$ M. A. Sentef, ${ }^{1}$ A. Georges $\odot,{ }^{2,3}$ T. Biesner, ${ }^{4}$ \\ E. Uykur, ${ }^{4}$ M. Dressel, ${ }^{4}$ A. Henderson, ${ }^{5}$ T. Siegrist, ${ }^{5}$ J. A. Schlueter ${ }^{5,6}$ K. Miyagawa $\odot,{ }^{7}$ K. Kanoda $\odot{ }^{7}$ \\ M.-S. Nam, ${ }^{8}$ A. Ardavan $\odot,{ }^{8}$ J. Coulthard, ${ }^{8}$ J. Tindall, ${ }^{8}$ F. Schlawin $\odot,{ }^{8}$ D. Jaksch, ${ }^{8}$ and A. Cavalleri $\odot^{1,8,7}$ \\ ${ }^{1}$ Max Planck Institute for the Structure and Dynamics of Matter, 22761 Hamburg, Germany \\ ${ }^{2}$ Center for Computational Quantum Physics (CCQ), The Flatiron Institute, New York, New York 10010, USA \\ ${ }^{3}$ Collège de France, 11 place Marcelin Berthelot, 75005 Paris, France \\ ${ }^{4}$ 1. Physikalisches Institut, Universität Stuttgart, 70569 Stuttgart, Germany \\ ${ }^{5}$ National High Magnetic Field Laboratory, \\ 1800 East Paul Dirac Drive, Tallahassee, Florida 32310, USA \\ ${ }^{6}$ Division of Material Research, National Science Foundation, Alexandria, Virginia 22314, USA \\ ${ }^{7}$ Department of Applied Physics, University of Tokyo, 7-3-1 Hongo, Bunkyo-ku, Tokyo 113-8656, Japan \\ ${ }^{8}$ Department of Physics, Clarendon Laboratory, University of Oxford, Oxford OX1 3PU, United Kingdom
}

(Received 27 April 2020; revised 4 June 2020; accepted 5 June 2020; published 6 August 2020)

\begin{abstract}
The properties of organic conductors are often tuned by the application of chemical or external pressure, which change orbital overlaps and electronic bandwidths while leaving the molecular building blocks virtually unperturbed. Here, we show that, unlike any other method, light can be used to manipulate the local electronic properties at the molecular sites, giving rise to new emergent properties. Targeted molecular excitations in the charge-transfer salt $\kappa$-(BEDT-TTF $)_{2} \mathrm{Cu}\left[\mathrm{N}(\mathrm{CN})_{2}\right] \mathrm{Br}$ induce a colossal increase in carrier mobility and the opening of a superconducting optical gap. Both features track the density of quasiparticles of the equilibrium metal and can be observed up to a characteristic coherence temperature $T^{*} \simeq 50 \mathrm{~K}$, far higher than the equilibrium transition temperature $T_{C}=12.5 \mathrm{~K}$. Notably, the large optical gap achieved by photoexcitation is not observed in the equilibrium superconductor, pointing to a light-induced state that is different from that obtained by cooling. First-principles calculations and model Hamiltonian dynamics predict a transient state with long-range pairing correlations, providing a possible physical scenario for photomolecular superconductivity.
\end{abstract}

DOI: $10.1103 /$ PhysRevX.10.031028

Subject Areas: Condensed Matter Physics, Strongly Correlated Materials, Superconductivity

Organic conductors based on the bisethylenedithiotetrathiafulvalene (BEDT-TTF) molecules display lowdimensional electronic structures and unconventional high- $T_{C}$ superconductivity [1-3]. In the crystals with a $\kappa$-type arrangement, the (BEDT $-\mathrm{TTF})^{+0.5}$ molecules (henceforth abbreviated as ET) form dimers, which are organized in planes on a triangular lattice [4]. Because of dimerization, the quarter-filled band originating from the overlap of the molecular orbitals splits into pairs of half-filled bands. The ET planes are separated by layers of monovalent anions, which act as charge reservoirs

*michele.buzzi@mpsd.mpg.de

† andrea.cavalleri@mpsd.mpg.de

Published by the American Physical Society under the terms of the Creative Commons Attribution 4.0 International license. Further distribution of this work must maintain attribution to the author(s) and the published article's title, journal citation, and DOI. Open access publication funded by the Max Planck Society.
[Fig. 1(a)]. The compound with the highest superconducting transition temperature is $\kappa-(\mathrm{ET})_{2} \mathrm{Cu}\left[\mathrm{N}(\mathrm{CN})_{2}\right] \mathrm{Br}$ (henceforth abbreviated as $\kappa-\mathrm{Br}$ ), for which $T_{C}=12.5 \mathrm{~K}$.

The low-energy physics of the $\kappa$-phase compounds is captured by a Hubbard model on a triangular lattice with weak and strong bonds [Fig. 1(a), lower-right panel] [5-7]. The ground state can be tuned either by applying hydrostatic pressure or by chemical substitution with different anions [8]. In both cases, the spacing between the dimers changes [Fig. 1(a)], directly acting on the hopping integrals $\left(t, t^{\prime}\right)$, leaving the on-site wave function and electronic correlations essentially unperturbed. Here, we explore the possibility of dynamically manipulating the Hamiltonian parameters and the many-body wave function by resonant excitation of ET molecular vibrations [Fig. 1(b)].

Equilibrium optical spectra were measured in the normal state of $\kappa$ - $\mathrm{Br}$ single crystals using a Fourier transform spectrometer for temperatures between $15 \mathrm{~K}$ and $300 \mathrm{~K}$ (see Fig. 2 and Supplemental Material [9]), in good agreement with those reported previously in the 
(a) Pressure control

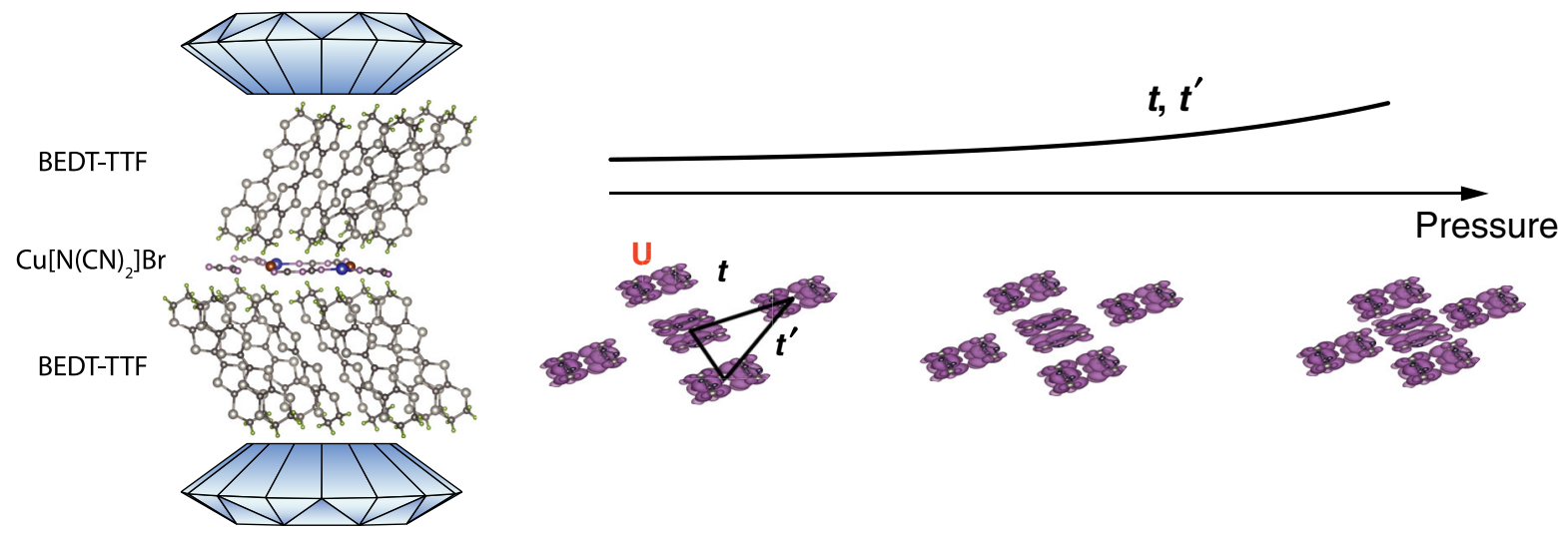

(b) Photomolecular control
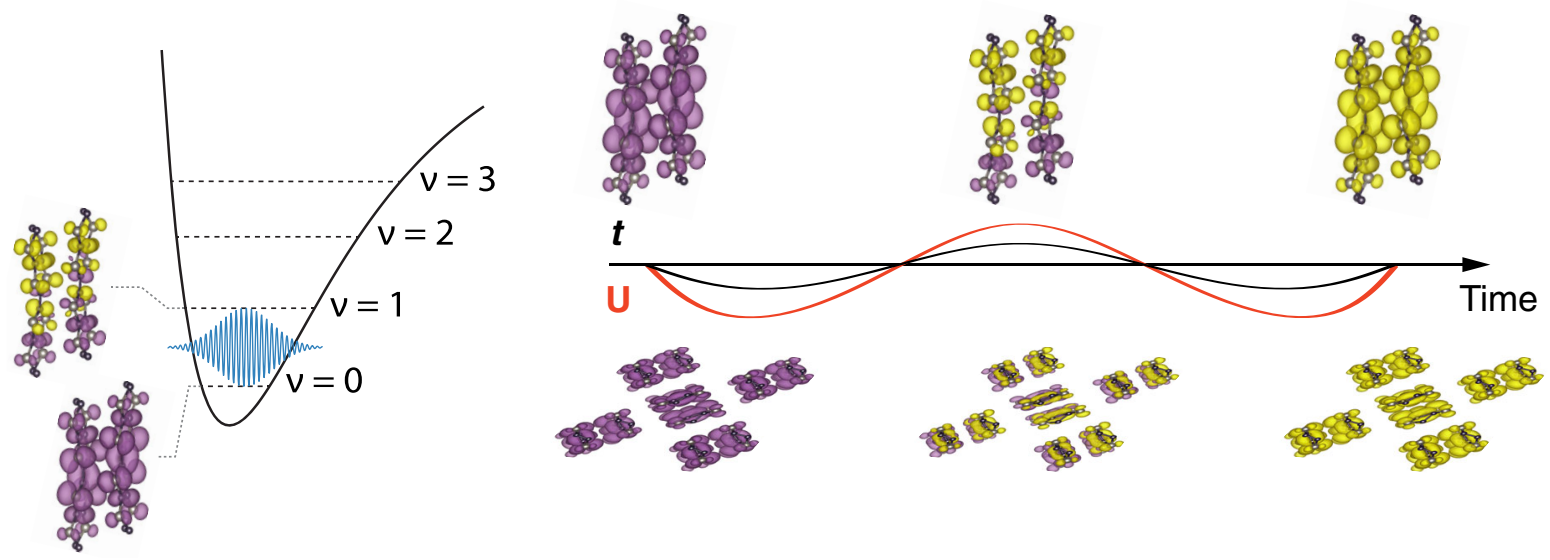

FIG. 1. (a) Left: Crystal structure of $\kappa-(\mathrm{BEDT}-\mathrm{TTF})_{2} \mathrm{Cu}\left[\mathrm{N}(\mathrm{CN})_{2}\right]$ Br. Alternating blocks of conducting and insulating layers are formed out of donor BEDT-TTF (abbreviated as ET) and polymeric chain-forming acceptor $\mathrm{Cu}\left[\mathrm{N}(\mathrm{CN})_{2}\right] \mathrm{Br}$ molecules, respectively. The ET molecules are paired in dimers. Right: View of the pressure-dependent arrangement of the dimers in the $\kappa$-phase (visualized along the long axis of the molecules, i.e., top view from left panel). As (external or chemical) pressure is applied, the spacing between dimers changes, thus acting on the transfer integrals $\left(t, t^{\prime}\right)$ and tuning the ground state of the material. (b) Left: Lennard-Jones potential, along with highest occupied molecular orbital (HOMO) plots for the first two vibrational levels. Violet and yellow colors denote the sign of the wave function. Right: Time evolution of the HOMO after pulsed excitation, creating a mixed population of $\nu=1$ and $\nu=0$ states, and the corresponding arrangement of the ET dimers (seen from the top). The periodic changes in the wave function introduce modulations in the on-dimer Hubbard $U$ and transfer integral $t$, opening new dynamic pathways for controlling the material properties.

literature [10,11]. In the equilibrium normal state $\left(T>T_{C}\right)$, $\kappa$-Br is a quasi-two-dimensional Fermi liquid, characterized by a narrow Drude peak in the in-plane optical conductivity, observed up to $T^{*} \simeq 50 \mathrm{~K}[12,13]$. For temperatures $T>T^{*}$, the quasiparticle response vanishes, and the material exhibits the behavior of a so-called "bad metal" [see Fig. 2(b)]. A large gap and a temperature-independent insulating optical conductivity are found along the perpendicular (interlayer) direction [14] [Fig. 2(a)]. Several narrow peaks are also evident in the midinfrared spectral region, corresponding to infrared-active vibrational modes of the ET molecules [15-17].
Femtosecond midinfrared pump pulses were polarized along the out-of-plane crystallographic axis and tuned to the spectral region where the molecular modes are found $\left(\nu_{\text {pump }} \simeq 900-2000 \mathrm{~cm}^{-1}, \lambda_{\text {pump }} \simeq 5-11 \mu \mathrm{m}\right)$. The peak pump electric field was varied between $500 \mathrm{kV} / \mathrm{cm}$ and $4 \mathrm{MV} / \mathrm{cm}$. The changes in low-frequency reflectivity and complex optical conductivity induced by midinfrared excitation were measured for frequencies between 0.8 and $7 \mathrm{THz}$, with probe pulses polarized along the conducting layers. These were detected by electro-optical sampling after reflection from the sample at different pump-probe time delays. 
(a)

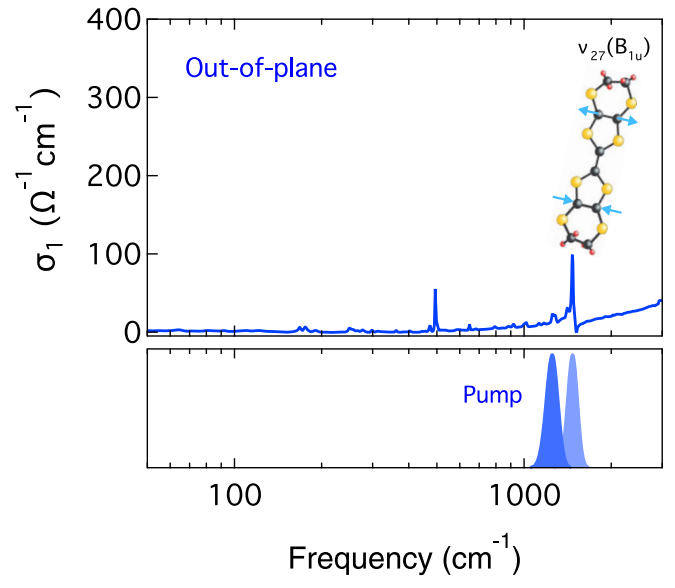

(b)

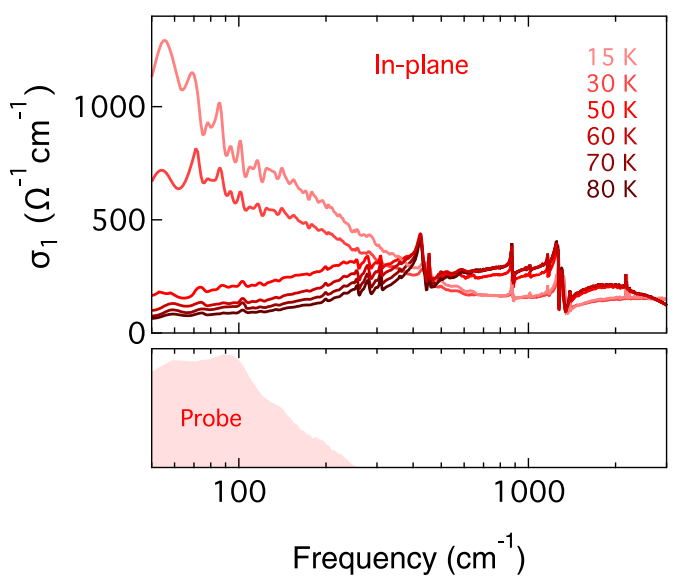

FIG. 2. (a) Upper panel: Out-of-plane equilibrium optical conductivity of $\kappa$-(ET) ${ }_{2} \mathrm{Cu}\left[\mathrm{N}(\mathrm{CN})_{2}\right] \mathrm{Br}$ measured at $T=15 \mathrm{~K}$ [14]. Lower panel: Midinfrared pump spectra tuned close to resonance with the $\nu_{27}$ molecular vibration. The eigenvectors corresponding to this mode are displayed in the upper panel [17]. (b) Upper panel: In-plane equilibrium optical conductivity measured at different temperatures between $15 \mathrm{~K}$ and $80 \mathrm{~K}$. Lower panel: Frequency spectrum of the THz probe pulses used in our experiment.

Figure 3 shows the most suggestive results discussed in this paper. Therein, optical properties $\left[R(\omega), \sigma_{1}(\omega)+i \sigma_{2}(\omega)\right]$ are reported for four representative base temperatures between $T=15 \mathrm{~K}$ and $T=70 \mathrm{~K}$, measured before (red) and 1 ps after photoexcitation (blue). Here, the pump pulses were tuned close to resonance with molecular vibrations corresponding to distortions of the $\mathrm{C}=\mathrm{C}$ bonds on the $\mathrm{ET}$ dimers (see sketch in Fig. 2). Importantly, these pump pulses, polarized along the insulating direction, penetrated deeper than the THz probe field (polarized along the metallic planes; see also Supplemental Material [9]). Hence, from the "raw" changes in electric field reflectance, $r_{1}(\omega)+i r_{2}(\omega)$, one could directly extract the complex optical conductivity, $\sigma_{1}(\omega)+i \sigma_{2}(\omega)$, without the need to account for an inhomogeneously excited medium [18-23].

The transient optical properties measured at base temperature $T=15 \mathrm{~K}$, that is, immediately above $T_{C}=12.5 \mathrm{~K}$, exhibited the largest changes. The reflectivity saturated to $R=1$ over a broad frequency range, decreasing only above $\sim 120 \mathrm{~cm}^{-1}$. Correspondingly, a gap opened in $\sigma_{1}(\omega)$, and a $1 / \omega$ divergence developed in $\sigma_{2}(\omega)$. A qualitatively similar response was recorded for $T=30 \mathrm{~K}$ and $T=50 \mathrm{~K}$, although with a smaller gap and $\sigma_{2}(\omega)$ divergence. For these three temperatures, the metallic optical spectra measured at negative time delays were fitted with a Drude-Lorentz model (red lines in Fig. 3) for normal conductors, whereas all the transient optical properties were fitted with an extension of the MattisBardeen model for superconductors of variable purity $[24,25]$ (blue lines in Fig. 3; see also Supplemental Material [9]).

A qualitatively different response was measured at $T=70 \mathrm{~K}$, for which $\sigma_{1}(\omega)$ increased rather than decreasing to zero, and $\sigma_{2}(\omega)$ remained characteristic of a metal without diverging toward low frequencies. These $70-\mathrm{K}$ spectra were then fitted with the same Drude-Lorentz model used to reproduce the equilibrium response.

For each of the three measurements at $T \leq 50 \mathrm{~K}$ reported in Fig. 3, we extracted the optical gap $2 \Delta$ from the Mattis-Bardeen fits. The temperature-dependent gap size could be fitted by the function $\Delta(T)=$ $\Delta(0) \tanh \left\{\beta \sqrt{\left[\left(T^{\prime}-T\right) / T\right]}\right\}$, where $2 \Delta(0)=23 \mathrm{meV}$ was the zero-temperature nonequilibrium gap, $\beta=1.74$, and $T^{\prime} \simeq 50 \mathrm{~K}$ provided an effective "critical" temperature for the nonequilibrium state [see Fig. 4(a)]. Note that $T^{\prime} \sim T^{*} \simeq 50 \mathrm{~K}$, which coincides with the temperature at which a coherent Drude peak was observed in the equilibrium metallic state of Fig. 2.

The connection between the superconducting-like optical properties measured in the transient state and the Fermi-liquid behavior of the equilibrium normal state $[13,26]$ is further underscored by the analysis reported in Fig. 4(b). Therein, the divergence in transient $\sigma_{2}(\omega)$ was used to estimate the light-induced "superfluid density," determined as $N_{\text {eff }}^{\text {Trans }}=\left(m V_{\text {Cell }} / e^{2}\right) \lim _{\omega \rightarrow 0}\left[\omega \sigma_{2}^{\text {Trans }}(\omega)\right]$. This quantity was compared to the temperaturedependent density of equilibrium quasiparticles, $N_{\mathrm{eff}}^{\text {Equil }}=$ $\left(m V_{\text {Cell }} / 4 \pi e^{2}\right)\left(\omega_{P}^{\text {Equil }}\right)^{2}$. Here, $m$ is the bare electron mass, $V_{\text {Cell }}$ the unit cell volume, $e$ the electron charge, and $\omega_{P}^{\text {Equil }}$ the equilibrium carrier plasma frequency (extracted from Drude-Lorentz fits). Even though the absolute values on the two vertical axes of Fig. 4(b) are different, the temperature dependences are similar, with the same onset at $T^{*} \simeq 50 \mathrm{~K}$.

The time dependence of these features is visualized in Fig. 5, where three different quantities are displayed. Figure 5(a) reports the transient integrated spectral weight $\int_{40 \mathrm{~cm}^{-1}}^{110 \mathrm{~cm}^{-1}} \sigma_{1}(\omega) d \omega$, which, in a superconductor, exhibits a depletion by an amount proportional to the density of 

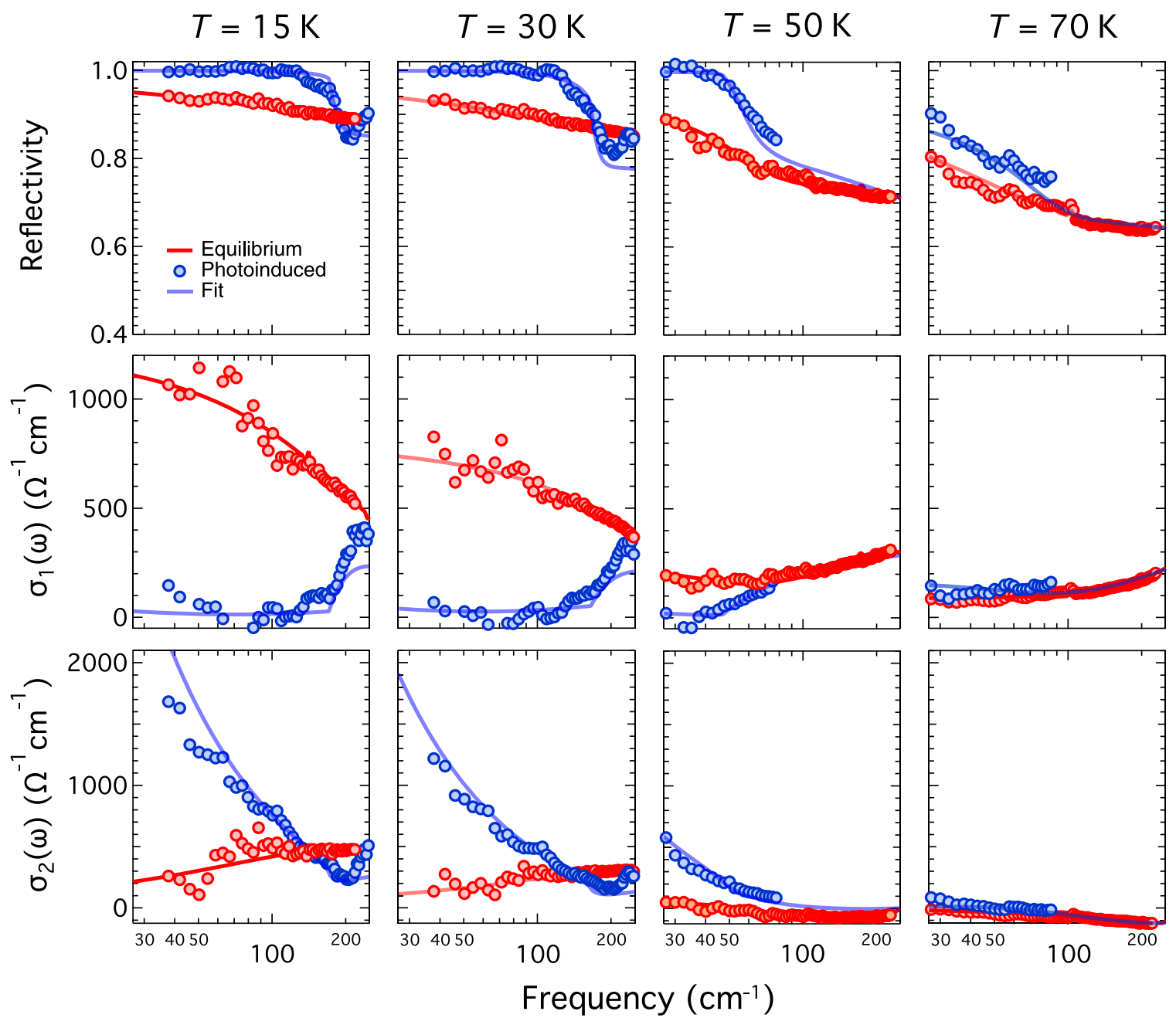

Frequency $\left(\mathrm{cm}^{-1}\right)$

FIG. 3. In-plane reflectivity, real and imaginary parts of the optical conductivity measured in $\kappa-(\mathrm{ET})_{2} \mathrm{Cu}\left[\mathrm{N}(\mathrm{CN})_{2}\right] \mathrm{Br}$ at equilibrium (red circles) and at $\tau \simeq 1 \mathrm{ps}$ time delay after vibrational excitation (blue circles), at four different base temperatures between $15 \mathrm{~K}$ and $70 \mathrm{~K}$. Lines are corresponding fits to the spectra. These fits were performed with a Drude-Lorentz model for the equilibrium response at all temperatures and for the transient spectra at $T=70 \mathrm{~K}$. A Mattis-Bardeen model for superconductors was used instead for the out-ofequilibrium response at $T \leq 50 \mathrm{~K}$. All data have been taken upon vibrational excitation close to resonance with the $\mathrm{C}=\mathrm{C}$ stretching mode $\left(\nu_{\text {pump }} \simeq 1250 \mathrm{~cm}^{-1}\right)$, with a pump fluence of $2 \mathrm{~mJ} / \mathrm{cm}^{2}$.

(a)

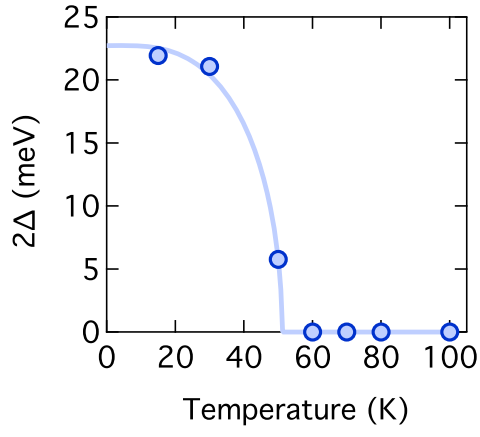

(b)

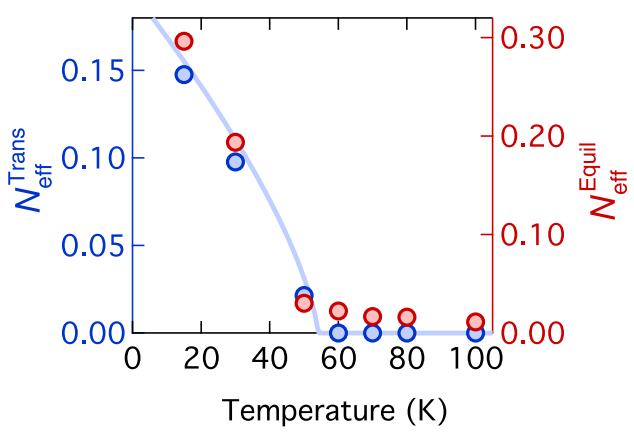

FIG. 4. (a) Photoinduced optical gap 2 $\Delta$, extracted from Mattis-Bardeen fits to the transient optical properties of Fig. 3, as a function of base temperature. (b) Blue, left scale: Temperature dependence of the effective number of "condensed" carriers per unit cell in the transient state, $N_{\text {eff }}^{\text {Trans }}$. Red, right scale: Corresponding effective number of mobile carriers per unit cell in the equilibrium metallic state before photoexcitation, $N_{\text {eff }}^{\text {Equil }}$ (see discussion in the text). These data have been taken upon vibrational excitation with a pump fluence of $2 \mathrm{~mJ} / \mathrm{cm}^{2}$. 

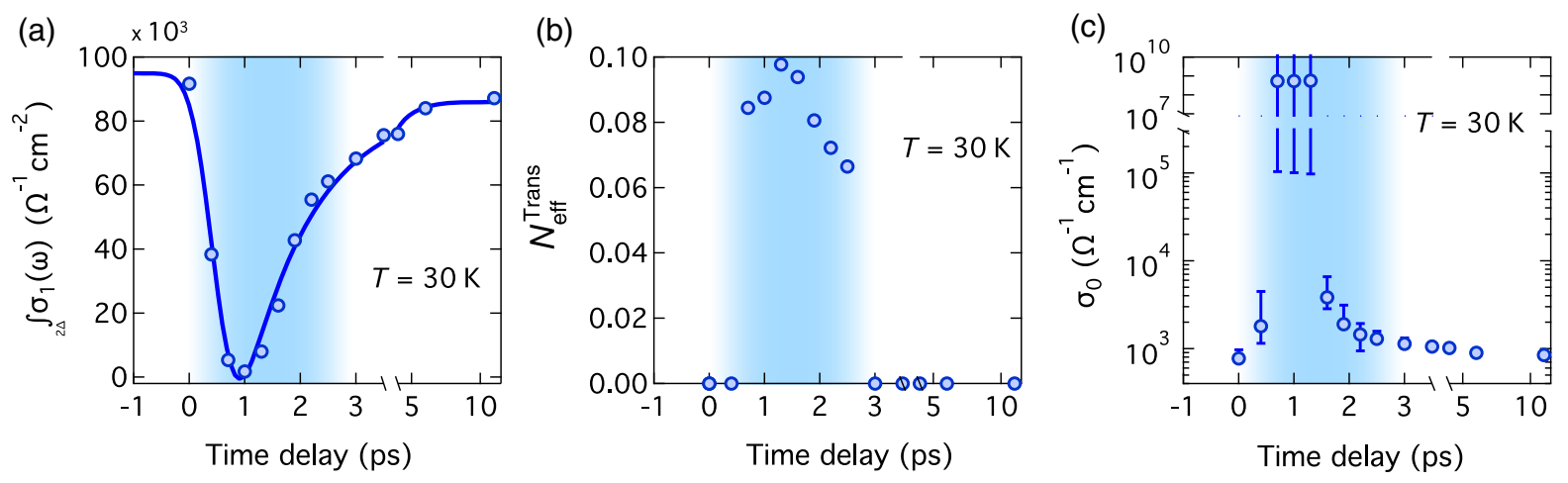

FIG. 5. (a) Dynamical evolution of the transient spectral weight in the real part of the optical conductivity as a function of pump-probe time delay. (b) Time evolution of the effective number of "condensed" carriers per unit cell in the transient state, $N_{\text {eff }}^{\text {Trans }}$. (c) Time evolution of the zero-frequency extrapolation of the optical conductivity, $\sigma_{0}=\left.\sigma_{1}(\omega)\right|_{\omega \rightarrow 0}$, extracted from Drude-Lorentz fits of the transient optical properties. Data have been taken at $T=30 \mathrm{~K}$ after vibrational excitation with a pump fluence of $2 \mathrm{~mJ} / \mathrm{cm}^{2}$.

condensed pairs. Figure 5(b) shows the transient superfluid density $N_{\text {eff }}^{\text {Trans }}$ extracted from the low-frequency imaginary conductivity, as in Fig. 4(b). Figure 5(c) reports $\sigma_{0}=\lim _{\omega \rightarrow 0} \sigma_{1}(\omega)$, that is, the extrapolated "zerofrequency" conductivity from Drude-Lorentz fits to the transient optical properties (see Supplemental Material [9]).
All three quantities changed promptly, immediately after photoexcitation, and relaxed within a few picoseconds, a timescale that we tentatively associate with the lifetime of the resonantly driven ET vibration. Importantly, $\sigma_{0}$ diverged to values larger than $10^{5} \Omega^{-1} \mathrm{~cm}^{-1}$ (corresponding to mean free paths of at least 100 unit cells), limited
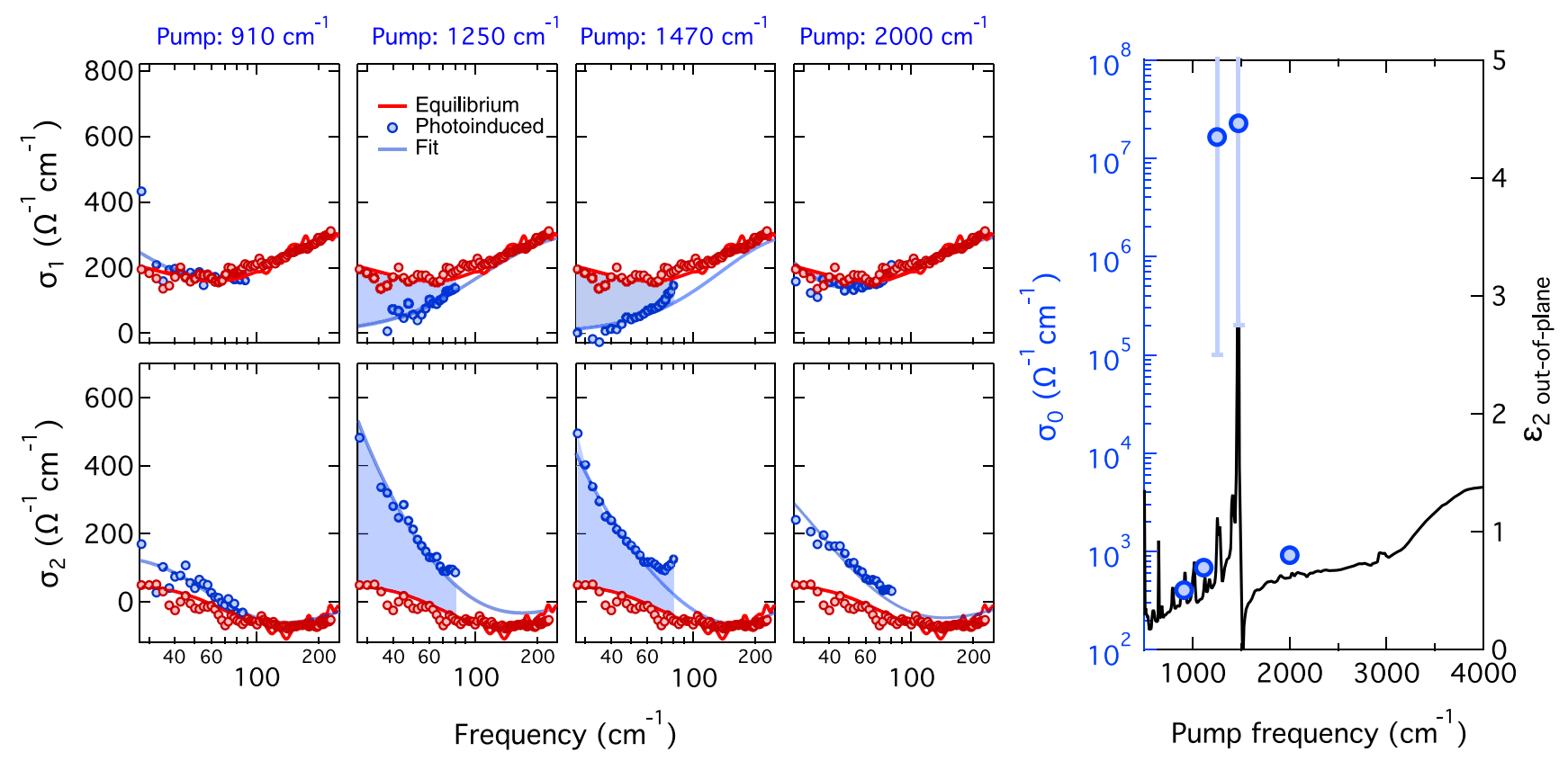

FIG. 6. Left panels: In-plane complex optical conductivity of $\kappa-(\mathrm{ET})_{2} \mathrm{Cu}\left[\mathrm{N}(\mathrm{CN})_{2}\right] \mathrm{Br}$ measured at $T=50 \mathrm{~K}$ in equilibrium (red circles) and at $\tau \simeq 1 \mathrm{ps}$ time delay after photoexcitation (blue circles) at four different pump frequencies: $\nu_{\text {pump }}=$ $910 \mathrm{~cm}^{-1}\left(\lambda_{\text {pump }}=11 \mu \mathrm{m}\right), \quad \nu_{\text {pump }}=1250 \mathrm{~cm}^{-1}\left(\lambda_{\text {pump }}=8 \mu \mathrm{m}\right), \quad \nu_{\text {pump }}=1470 \mathrm{~cm}^{-1}\left(\lambda_{\text {pump }}=6.8 \mu \mathrm{m}\right), \quad$ and $\quad \nu_{\text {pump }}=2000 \mathrm{~cm}^{-1}$ $\left(\lambda_{\text {pump }}=5 \mu \mathrm{m}\right)$. Lines are corresponding fits performed with a Drude-Lorentz model. The $\nu_{\text {pump }}=2000 \mathrm{~cm}^{-1}$ spectra display an enhancement of $\sigma_{2}(\omega)$ in the absence of a gap opening in $\sigma_{1}(\omega)$. This is indicative of a slight increase in metallicity, with no superconducting-like response. All data have been taken with a constant pump fluence of $2 \mathrm{~mJ} / \mathrm{cm}^{2}$. Right panel: Zero-frequency extrapolation of the transient optical conductivity, $\sigma_{0}=\left.\sigma_{1}(\omega)\right|_{\omega \rightarrow 0}$ (see main text), extracted from the spectra on the left for different pump wavelengths (blue circles, left scale). A data point at $\nu_{\text {pump }}=1100 \mathrm{~cm}^{-1}\left(\lambda_{\text {pump }}=9 \mu \mathrm{m}\right.$ ) is also reported, for which the transient spectra look identical to those at $\nu_{\text {pump }}=910 \mathrm{~cm}^{-1}$ (negligible pump-induced effect). The equilibrium out-of-plane imaginary dielectric function [14] is also displayed (black line, right scale) to visualize the resonant photoconductivity behavior on the $\mathrm{C}=\mathrm{C}$ ET vibration. 
here by the $0.8-\mathrm{THz}$ low-frequency cutoff of our measurement, which in turn was set by the relaxation time of the state ( 2 ps).

In Fig. 6, we report the transient complex optical conductivity after photoexcitation at different driving frequencies, all measured by keeping the pump fluence fixed to $2 \mathrm{~mJ} / \mathrm{cm}^{2}$. A superconducting-like response could only be observed for excitation at $8 \mu \mathrm{m}$ and $6.8 \mu \mathrm{m}$ [see also Fig. 1(a)], while detuned driving to both red $\left(\lambda_{\text {pump }}=11 \mu \mathrm{m}\right.$, resonant to a weaker ET vibration) and blue $\left(\lambda_{\text {pump }}=5 \mu \mathrm{m}\right)$ sides resulted in a slight increase in $\sigma_{2}(\omega)$ and negligible changes in $\sigma_{1}(\omega)$. The $\sigma_{0}$ values extracted from these data are displayed in the right panel of Fig. 6, along with the equilibrium outof-plane imaginary dielectric function. This plot confirms that superconducting-like optical properties in the transient state can only be observed when pumping in the 6.8 $-8 \mu \mathrm{m}$ range, close to resonance with $\mathrm{C}=\mathrm{C}$ modes of the ET molecule.

In searching for a microscopic mechanism for the observed photomolecular response, we note that in a previous work on the one-dimensional Mott insulator
ET- $\mathrm{F}_{2} \mathrm{TCNQ}$, featuring the same ET molecular building block as $\kappa$-Br, selective driving of an IR-active vibration was shown to provide a dynamical modulation of the onsite Hubbard- $U$ interaction, achieved by quadratic electronphonon coupling $[27,28]$.

In the $\kappa$ - $\mathrm{Br}$ system studied here, ab initio calculations in the frozen-phonon approximation yielded an estimate for the on-dimer Hubbard $U$, hopping matrix elements $t$ (strong bonds), and $t^{\prime}$ (weak bonds) as a function of normal mode distortion (see Supplemental Material [9]). Excitation of the molecular $\mathrm{C}=\mathrm{C}$ stretching modes (i.e., the $\nu_{27}$ vibration at $6.8 \mu \mathrm{m}$ ) leads to a significant modulation of $U$ and $t$, whereas $t^{\prime}$ remains mostly unaffected [Fig. 7(a) and Supplemental Material [9] ]. By contrast, excitation of molecular modes that involve mainly motions in the terminal ethylene groups of the ET molecule (i.e., at 9 and $11 \mu \mathrm{m}$ ) does not produce any sizable change in the effective electronic parameters [Fig. 7(b)].

Starting from these estimates, we performed simulations of a driven Fermi-Hubbard model on a triangular ladder system (see Supplemental Material [9] for the Hamiltonian and specific geometry of the system). In the driven state,

(a) $\mathrm{C}=\mathrm{C}$ stretching mode $\left(1470 \mathrm{~cm}^{-1}\right)$
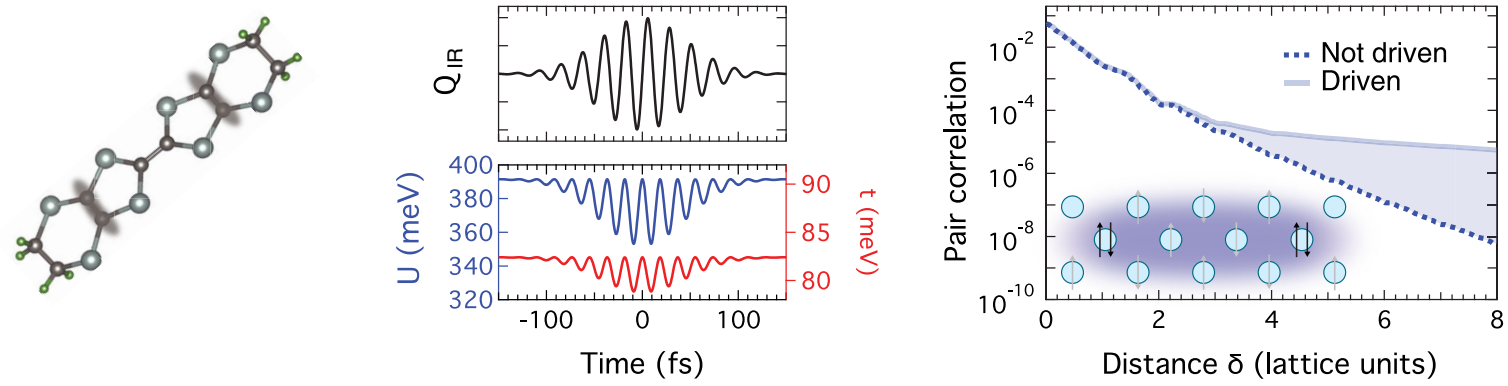

(b) Terminal ethylene mode $\left(920 \mathrm{~cm}^{-1}\right)$
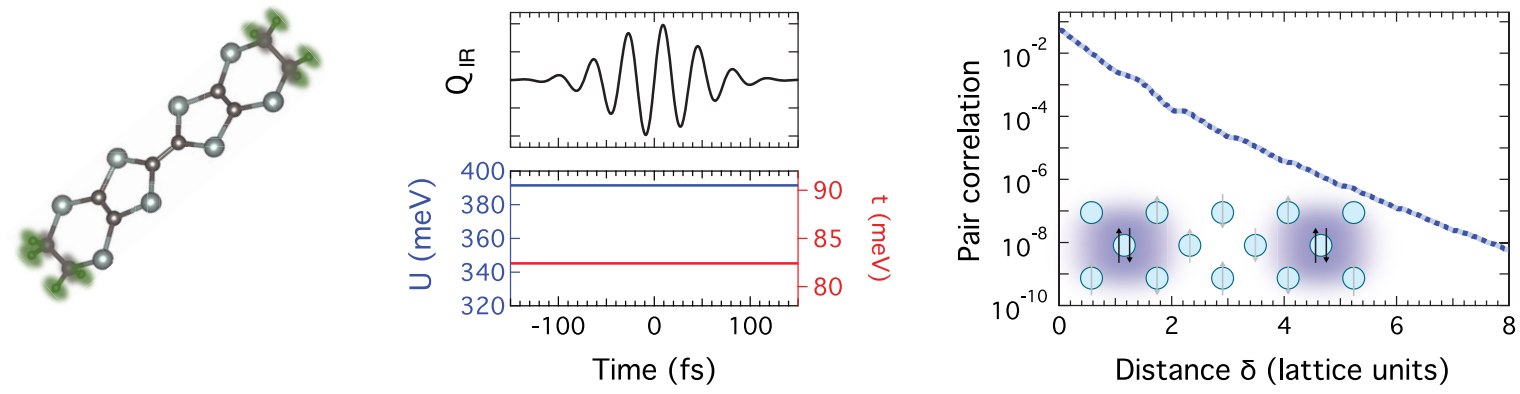

FIG. 7. Left panels: Atomic motions corresponding to the $1470 \mathrm{~cm}^{-1}$ (a) and $920 \mathrm{~cm}^{-1}$ (b) modes of the ET molecule. Center panels: Time-dependent displacement along the normal coordinates and induced modulations of the on-site Hubbard $U$ interaction and hopping matrix element $t$, calculated with ab initio simulations in the frozen-phonon approximation for the two different driven modes. Right panels: Corresponding spatial evolution of the pair correlation function, calculated with a time-dependent triangular ladder FermiHubbard model in the undriven case (blue dashed line) and driven case (blue solid lines) for the two modes. The insets display a schematic representation of the correlation between pairs on different sites. 
correlations between pairs of doublons residing on different sites were quantified by the doublon correlation function $d_{i}^{\dagger} d_{j}=c_{i \uparrow}^{\dagger} c_{i \downarrow}^{\dagger} c_{j \downarrow} c_{j \uparrow}$ (here, $c_{i \sigma}^{\dagger}$ and $c_{i \sigma}$ are single-particle creation and annihilation operators, respectively). In the simulations, the system was initialized in its half-filled zero-temperature ground state, yielding doublon correlations that decay exponentially with distance [blue dashed line in Fig. 7(a)]. When the driving was turned on, longrange, uniform on-site doublons were formed with long correlation distances. These correlations stabilized and persisted for timescales compatible with those explored in the experiment [blue solid line, Fig. 7(a); see also Supplemental Material [9] ]. When considering these theoretical predictions, a driving-induced emergence of longrange order in the system is hypothesized, where the external driving establishes both a large density of on-site doublons and phase coherence over macroscopic distances. The predictions reported in Fig. 7(b) for excitation of a terminal ethylene mode, for which no enhancement in coherent on-site doublons is expected, are in agreement with the experimental results of Fig. 6, which show that a coherent state is induced only for excitation of the $\mathrm{C}=\mathrm{C}$ stretching mode.

The scenario proposed by the model sketched in Fig. 7 is notable as it implies the formation of a qualitatively new state of the system, with double occupancies that are "synchronized" through the $U / t$ modulation. This mechanism, which may explain the appearance of a large gap not present in the low-temperature equilibrium superconductor, bears some resemblance to the proposed photoinduced $\eta$ - pair superconductor [29-31], in which repulsive pairs are activated by the drive.

Other mechanisms, different from the one discussed here, may also be at play [32-34], including the stabilization of preexisting Cooper pairs in the normal state [35], which have been revealed by superconducting Nernst effect measurements [36]. Finally, the possible appearance of a negative- $U$ superconductivity through electronic squeezing in a molecular excited state should also be considered, especially in the context of a nearly frustrated electronic structure like the present one [37,38].

The research leading to these results received funding from the European Research Council under the European Union's Seventh Framework Programme (FP7/20072013)/ERC Grant Agreement No. 319286 (QMAC). We acknowledge support from the Deutsche Forschungsgemeinschaft (DFG) via the Cluster of Excellence "The Hamburg Centre for Ultrafast Imaging" (EXC 1074Project ID No. 194651731), the priority program SFB925, and the Emmy Noether program (SE 2558/2-1). J.A.S. acknowledges support from the Independent Research/Development program while serving at the National Science Foundation. K. M. and K. K. acknowledge support from the Japan Society for the Promotion of
Science Grant No. 18H05225. T. S. and A. H. acknowledge funding from the NSF under Grant No. DMR-1534818. The National High Magnetic Field Laboratory is supported by the NSF under Grant No. DMR-1644779 and the State of Florida.

[1] T. Ishiguro, K. Yamaji, and G. Saito, Organic Superconductors, 2nd ed. (Springer-Verlag Berlin Heidelberg, 1998).

[2] D. Jérome, The Physics of Organic Superconductors, Science 252, 1509 (1991).

[3] M. Lang and J. Mueller, Organic Superconductors, arXiv: cond-mat/0302157.

[4] A. Ardavan, S. Brown, S. Kagoshima, K. Kanoda, K. Kuroki, H. Mori, M. Ogata, S. Uji, and J. Wosnitza, Recent Topics of Organic Superconductors, J. Phys. Soc. Jpn. 81, 011004 (2012).

[5] A. Fortunelli and A. Painelli, On the Ab Initio Evaluation of Hubbard Parameters. II. The $\kappa$-(BEDT-TTF $)_{2}$ $\mathrm{Cu}\left[\mathrm{N}(\mathrm{CN})_{2}\right]$ Br Crystal, J. Chem. Phys. 106, 8051 (1997).

[6] H. C. Kandpal, I. Opahle, Y.Z. Zhang, H. O. Jeschke, and R. Valentí, Revision of Model Parameters for $\kappa$-Type Charge Transfer Salts: An Ab Initio Study, Phys. Rev. Lett. 103, 067004 (2009).

[7] R. H. McKenzie, A Strongly Correlated Electron Model for the Layered Organic Superconductors $\kappa$-(BEDT-TTF $)_{2} \mathrm{X}$, Comments Condens. Matter Phys. 18, 309 (1998), https:// arxiv.org/abs/cond-mat/9802198.

[8] H. Kino and H. Fukuyama, Phase Diagram of TwoDimensional Organic Conductors: (BEDT-TTF $)_{2} \mathrm{X}$, J. Phys. Soc. Jpn. 65, 2158 (1996).

[9] See Supplemental Material at http://link.aps.org/ supplemental/10.1103/PhysRevX.10.031028 for sample preparation, determination of equilibrium and transient optical properties, fitting models, extended data sets, and $a b$ initio calculations.

[10] J. E. Eldridge, K. Kornelsen, H. H. Wang, J. M. Williams, A. V.S. Crouch, and D. M. Watkins, Infrared Optical Properties of the $12 \mathrm{~K}$ Organic Superconductor $\kappa-(\mathrm{BEDT}-\mathrm{TTF})_{2} \mathrm{Cu}\left[\mathrm{N}(\mathrm{CN})_{2}\right] \mathrm{Br}$, Solid State Commun. 79, 583 (1991).

[11] D. Faltermeier, J. Barz, M. Dumm, M. Dressel, N. Drichko, B. Petrov, V. Semkin, R. Vlasova, C. Meźière, and P. Batail, Bandwidth-Controlled Mott Transition in $\kappa$-(BEDT-TTF $)_{2} \mathrm{Cu}\left[\mathrm{N}(\mathrm{CN})_{2}\right] \mathrm{Br}_{x} \mathrm{Cl}_{1-x}$ : Optical Studies of Localized Charge Excitations, Phys. Rev. B 76, 165113 (2007).

[12] M. Dumm, D. Faltermeier, N. Drichko, M. Dressel, C. Mézière, and P. Batail, Bandwidth-Controlled Mott Transition in $\kappa$-(BEDT-TTF $)_{2} \mathrm{Cu}\left[\mathrm{N}(\mathrm{CN})_{2}\right] \mathrm{Br}_{x} \mathrm{Cl}_{1-x}$ : Optical Studies of Correlated Carriers, Phys. Rev. B 79, 195106 (2009).

[13] M. Dressel, M. Dumm, N. Drichko, C. Méziere, and P. Batail, Coherent Particle Response of Two-Dimensional Organic Conductors upon Crossing the Mott Transition, J. Phys. Conf. Ser. 200, 012029 (2010).

[14] J. J. McGuire, T. Rõõm, A. Pronin, T. Timusk, J. A. Schlueter, M.E. Kelly, and A. M. Kini, Incoherent 
Interplane Conductivity of $\kappa-(\mathrm{BEDT}-\mathrm{TTF})_{2} \mathrm{Cu}\left[\mathrm{N}(\mathrm{CN})_{2}\right] \mathrm{Br}$, Phys. Rev. B 64, 094503 (2001).

[15] J. E. Eldridge, C. C. Homes, J. M. Williams, A. M. Kini, and H. H. Wang, The Assignment of the Normal Modes of the BEDT-TTF Electron-Donor Molecule Using the Infrared and Raman Spectra of Several Isotopic Analogs, Spectrochim. Acta, Part A 51, 947 (1995).

[16] J. E. Eldridge, Y. Xie, H. H. Wang, J. M. Williams, A. M. Kini, and J. A. Schlueter, Electron-Phonon Effects in the Organic Superconductor $\kappa-(\mathrm{BEDT}-\mathrm{TTF})_{2} \mathrm{Cu}\left[\mathrm{N}(\mathrm{CN})_{2}\right] \mathrm{Br}$, Spectrochim. Acta, Part A 52, 45 (1996).

[17] M. Maksimuk, K. Yakushi, H. Taniguchi, K. Kanoda, and A. Kawamoto, The $\mathrm{C}=\mathrm{C}$ Stretching Vibrations of $\kappa$-(BEDT-TTF $)_{2} \mathrm{Cu}\left[\mathrm{N}(\mathrm{CN})_{2}\right] \mathrm{Br}$ and Its Isotope Analogues, J. Phys. Soc. Jpn. 70, 3728 (2001).

[18] D. Fausti, R. I. Tobey, N. Dean, S. Kaiser, A. Dienst, M. C. Hoffmann, S. Pyon, T. Takayama, H. Takagi, and A. Cavalleri, Light-Induced Superconductivity in a StripeOrdered Cuprate, Science 331, 189 (2011).

[19] W. Hu, S. Kaiser, D. Nicoletti, C. R. Hunt, I. Gierz, M. C. Hoffmann, M. Le Tacon, T. Loew, B. Keimer, and A. Cavalleri, Optically Enhanced Coherent Transport in $\mathrm{YBa}_{2} \mathrm{Cu}_{3} \mathrm{O}_{6.5}$ by Ultrafast Redistribution of Interlayer Coupling, Nat. Mater. 13, 705 (2014).

[20] S. Kaiser et al. Optically Induced Coherent Transport Far above $T_{c}$ in Underdoped $\mathrm{YBa}_{2} \mathrm{Cu}_{3} \mathrm{O}_{6+\delta}$, Phys. Rev. B 89, 184516 (2014).

[21] M. Mitrano et al., Possible Light-Induced Superconductivity in $K_{3} \mathrm{C}_{60}$ at High Temperature, Nature (London) 530, 461 (2016).

[22] D. Nicoletti, E. Casandruc, Y. Laplace, V. Khanna, C. R. Hunt, S. Kaiser, S. S. Dhesi, G. D. Gu, J.P. Hill, and A. Cavalleri, Optically Induced Superconductivity in Striped $\mathrm{La}_{2-x} \mathrm{Ba}_{x} \mathrm{CuO}_{4}$ by Polarization-Selective Excitation in the Near Infrared, Phys. Rev. B 90, 100503 (2014).

[23] A. Cantaluppi, M. Buzzi, G. Jotzu, D. Nicoletti, M. Mitrano, D. Pontiroli, M. Riccò, A. Perucchi, P. Di Pietro, and A. Cavalleri, Pressure Tuning of Light-Induced Superconductivity in $K_{3} C_{60}$, Nat. Phys. 14, 837 (2018).

[24] W. Zimmermann, E. H. Brandt, M. Bauer, E. Seider, and L. Genzel, Optical Conductivity of BCS Superconductors with Arbitrary Purity, Physica C (Amsterdam) 183, 99 (1991).
[25] M. Dressel and G. Grüner, Electrodynamics of Solids (Cambridge University Press, Cambridge, England, 2002).

[26] A. Kawamoto, K. Miyagawa, Y. Nakazawa, and K. Kanoda, ${ }^{13}$ C NMR Study of Layered Organic Superconductors Based on BEDT-TTF Molecules, Phys. Rev. Lett. 74, 3455 (1995).

[27] S. Kaiser et al., Optical Properties of a Vibrationally Modulated Solid State Mott Insulator, Sci. Rep. 4, 3823 (2015).

[28] R. Singla et al., THz-Frequency Modulation of the Hubbard $U$ in an Organic Mott Insulator, Phys. Rev. Lett. 115, 187401 (2015).

[29] F. Peronaci, O. Parcollet, and M. Schirò, Enhancement of Local Pairing Correlations in Periodically Driven Mott Insulators. arXiv:1904.00857 (2019).

[30] J. Tindall, B. Buča, J. R. Coulthard, and D. Jaksch, HeatingInduced Long-Range $\eta$ Pairing in the Hubbard Model, Phys. Rev. Lett. 123, 030603 (2019).

[31] J. Li, D. Golez, P. Werner, and M. Eckstein, Long-Range $\eta$-Pairing in Photodoped Mott Insulators, arXiv:1908 .08693 .

[32] A. Nava, C. Giannetti, A. Georges, E. Tosatti, and M. Fabrizio, Cooling Quasiparticles in $A_{3} C_{60}$ Fullerides by Excitonic Midinfrared Absorption, Nat. Phys. 14, 154 (2018).

[33] H. Dehghani, Z. M. Raines, V. M. Galitski, and M. Hafezi, Optical Enhancement of Superconductivity via Targeted Destruction of Charge Density Waves, Phys. Rev. B 101, 224506 (2020).

[34] A. Sheikhan and C. Kollath, Dynamically Enhanced Unconventional Superconducting Correlations in a Hubbard Ladder, arXiv:1902.07947.

[35] Y. J. Uemura, Dynamic Superconductivity Responses in Photoexcited Optical Conductivity and Nernst Effect, Phys. Rev. Mater. 3, 104801 (2019).

[36] M. S. Nam, A. Ardavan, S. J. Blundell, and J. A. Schlueter, Fluctuating Superconductivity in Organic Molecular Metals Close to the Mott Transition, Nature (London) 449, 584 (2007).

[37] D. M. Kennes, E. Y. Wilner, D. R. Reichman, and A. J. Millis, Transient Superconductivity from Electronic Squeezing of Optically Pumped Phonons, Nat. Phys. 13, 479 (2017).

[38] M. A. Sentef, Light-Enhanced Electron-Phonon Coupling from Nonlinear Electron-Phonon Coupling, Phys. Rev. B 95, 205111 (2017). 